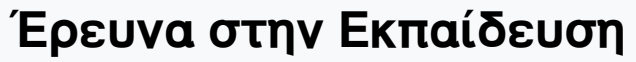

Tóp. 3 (2015)

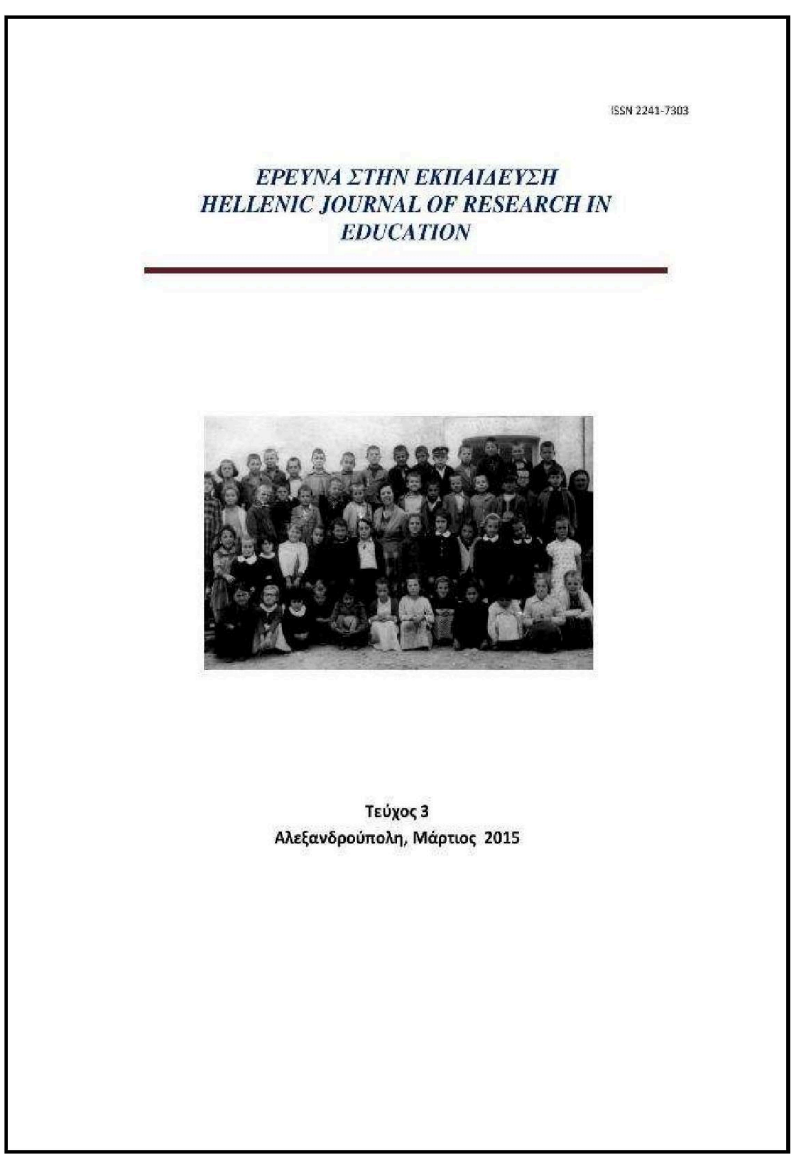

Etre instituteur de maternelle en Grèce :

Représentations et choix du métier

Stathis Balias, Pandelis Kiprianos

doi: $10.12681 /$ hjre.8847

Copyright @ 2016, Stathis Balias, Pandelis Kiprianos

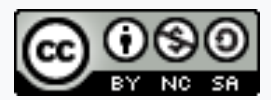

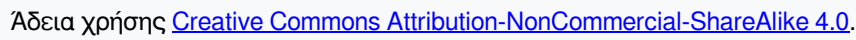

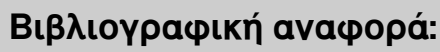

Balias, S., \& Kiprianos, P. (2015). Etre instituteur de maternelle en Grèce : Représentations et choix du métier. 'E $\rho \varepsilon u v a$

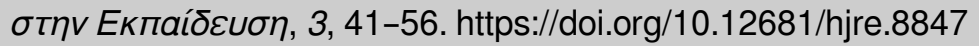




\title{
Etre instituteur de maternelle en Grèce : Représentations et choix du métier
}

\author{
STATHIS BALIAS, professeur adjoint - PANDELIS KIPRIANOS, professeur
}

\author{
balias@upatras.gr \\ kiprian@upatras.gr
}

\begin{abstract}
Résumé
La représentation sociale d'un métier participe à la fixation de sa valeur et engendre des pratiques. Partant de cette considération nous allons examiner, sous un angle comparatif, les représentations et le choix du métier de l'instituteur de la maternelle en Grèce. Pour cela nous nous appuierons sur trois indices: la position institutionnelle de l'instituteur, la demande sociale du métier et la composition sociale du corps des instituteurs. Pour donner une assise comparative à notre argumentation, nous allons nous référer, dans un premier temps, à des études relatives dans d'autres pays. Ensuite, nous nous esquisserons un aperçu de l'évolution historique de l'institution en Grèce en fonction de ses objectifs pédagogiques et de son public. Enfin, appuyés essentiellement sur des archives, des rapports d'inspection et des données statistiques nous nous tenterons de sonder la demande du métier et la composition sociale des instituteurs de maternelle. La revalorisation des études et du diplôme de l'instituteur depuis notamment 1982, nous arguons, a été suivie d'une certaine amélioration de sa représentation sociale. Cela se reflète sur la demande du métier et sur la composition sociale des instituteurs.
\end{abstract}

Keywords: Social Representation, Preschool teacher, Greece, Profession, Comparison

\section{Introduction}

La représentation sociale d'un métier participe à la fixation de sa valeur et engendre des choix et des pratiques. A leur tour, ces choix et pratiques pèsent sur la représentation du métier et sa valeur. Partant de cette considération nous allons examiner, sous un angle comparatif, les représentations et le choix du métier de l'instituteur de la maternelle en 
Grèce. Pour cela nous nous appuierons sur trois indices: la position institutionnelle de l'instituteur, la demande sociale du métier et la composition sociale du corps des instituteurs.

Notre hypothèse est la suivante: Pendant longtemps malgré une rhétorique discordante l'institutrice en tant que métier était peu valorisée. Cela se reflétait sur ses origines sociales modestes. La revalorisation des études et du diplôme depuis notamment 1982, allant de pair avec l'ouverture au sexe masculin, a été suivie d'un certain changement de sa représentation sociale. Cela se reflète sur la demande du métier et sur la composition sociale des instituteurs.

Pour étayer note hypothèse nous procédons sur trois étapes. Pour donner une assise comparative à notre argumentation, nous allons nous référer, dans un premier temps, à des études relatives menées dans d'autre pays. Ensuite, pour montrer l'évolution diachronique du métier nous nous esquisserons un bref aperçu historique de l'institution en Grèce en fonction de ses objectifs pédagogiques et de son public. Enfin, appuyés essentiellement sur des archives, des rapports d'inspection et des données statistiques nous nous tenterons de sonder la demande du métier et la composition sociale des instituteurs de maternelle.

\section{Représentations de l'enfance et de la maternelle}

La représentation ménage les rapports entre sujets et objets et génère des pratiques des acteurs. B. Bernstein et D. Young (1973, pp. 13-23) ont montré comment la culture des couches moyennes telle qu'elle transparaît dans la conception du jeu imprègne les pédagogies suivies dans la maternelle. D'autres auteurs, sociologues et psychologues parviennent à des constats similaires. J. Bruner (1996, pp. 72-73) voit dans la conception et la mise en œuvre du Head Start aux Etats-Unis, ${ }^{1}$ I'empreinte des valeurs des couches moyennes sur l'enfance et le traite, entre autres, comme un véhicule de diffusion dans les milieux ethniques et populaires.

J. C. Champoredon et J. Prévot (1973, p. 319) font état d'une «définition nouvelle de la prime enfance». "On peut distinguer semble-t-il », ils écrivent, "deux grandes principes d'évolution: I'un conduit à la 'libération' de l'enfant à l'égard des contraintes et

\footnotetext{
${ }^{1}$ Le programme américain, symbole de l'éducation compensatoire, s'adresse principalement aux afro-américains et aux hispaniques dont la fréquentation de la maternelle est faible.
} 
l'enrégimentement rigoureux de la 'salle d'asile', l'autre à l'autonomisation croissante de la maternelle par rapport à l'enseignement primaire». Signalons encore l'analyse d'E. Plaisance sur la maternelle en France. II distingue deux modèles pédagogiques, un premier nommé productif, en cours après la seconde guerre mondiale et centré sur la «connaissance» et un seconde, après 1968, expressif, reposant sur l' "expression de sa propre personnalité» (1986, pp. 137-8).

Considéré sous cet angle, le contenu pédagogique de la maternelle, pèse sur la perception de l'institution, sur sa demande sociale et son public. Plus ce contenu est familier aux valeurs d'une couche sociale plus elle est susceptible de lui plaire. Sous cet angle nous pouvons appréhender la mutation du public des maternelles en France après la deuxième guerre mondiale. «Contrairement à la situation présentée en 1954», conclut E. Plaisance, "la répartition spatiale de la préscolarisation et celle de la population ouvrière ne se superposent plus» $(1986$, p. 61). Il est fort possible qu'une évolution analogue du public de la maternelle ait eu lieu dans d'autres pays après 1968 comme conséquence des divers changements y compris de la pédagogie en cours. Cela pourrait valoir aussi bien pour l'Italie ainsi que pour l'Allemagne Fédérale qui après 1968 adoptent des pédagogies plus en accord avec l'esprit du temps favorisant l'expression personnelle et le travail en groupes (Konrad, 2004, pp. 192-199).

L'évolution signalée du public de la maternelle, des couches populaires vers les couches plus aisées, ne constitue, certes, pas le modèle - type dans l'histoire de l'institution. Aux Etats-Unis, jusqu' dans les années 1960, dans une maternelle peu fréquentée, le taux des enfants de couleur et hispaniques est nettement inférieur à celui des enfants blancs, notamment de condition socio-économique moins modeste. Malgré le lancement du programme Head Start qui a bénéficié surtout aux Afro-américains, au début des années '90, le taux des enfants de 3-5 ans inscrits en crèche (center-basedchild care programs) s'élevait à 55 pour les noirs, 43 pour les blancs et 32\% pour les hispaniques (Liang, 1998, p. 7).

Pour nous faire une image plus globale nous mentionnons une enquête internationale menée dans les années 1980 par l'IEA (International Association for the Evaluation of Educational Achievement. «Lorsque l'on interroge les mères sur les raisons qui les poussent à conduire leur enfant dans un centre d'éducation préscolaire " on y lit, " certaines soulignent l'intérêt pour le développement psychologique de l'enfant, tandis que d'autres invoquent pour première raison des problèmes de garde ou leur occupation 
professionnelle» (Commission, 1995, p. 9). Le tableau 1 indique, la répartition des réponses par pays.

$\begin{array}{cccc}\text { Pays } & \begin{array}{c}\text { Tableau 1. Perception de la maternelle par des parents dans certains pays* } \\ \text { Bénéfice psychologique } \\ \text { pour l'enfant }\end{array} & \begin{array}{c}\text { Autres raisons } \\ \text { Problème de garde }\end{array} \\ \text { Hong-Kong } & 100 & 0 & 0 \\ \text { Belgique } & 75 & 24 & 1 \\ \text { Italie } & 76 & 23 & 1 \\ \text { Espagne } & 64 & 36 & 0 \\ \text { Chine } & - & - & - \\ \text { USA } & 44 & 49 & 7 \\ \text { Finlande } & 19 & 80 & 1,2 \\ \text { Nigéria } & 28 & 67 & 4,6 \\ \text { Portugal } & 29 & 57 & 14,2 \\ \text { Thailande } & 20 & 80 & 0,4\end{array}$

*Source: Commission, 1995, p. 9.

Les experts de la Task Force Ressources Humaines, Education, Formation et Jeunesse de la Commission Européenne en tirent la conclusion suivante: «Les données sont claires: dans les pays caractérisés par un taux élevé de fréquentation d'un centre d'éducation préscolaire, le pourcentage de mères soulignant la valeur éducative de ces structures d'accueil est élevé. A l'inverse, dans les pays caractérisés par un faible taux de fréquentation de ces centres d'éducation préscolaire, les mères qui y ont recours soulignent davantage leur problème de garde. La situation varie donc d'un pays à l'autre». (Commission, 1995, p. 10). En d'autres termes, la variable explicative retenue est celle de la tradition et du vécu en la matière.

L'interprétation nous semble fondée. Les premiers pays du tableau, notamment les trois européens, disposent d'une forte tradition dans le domaine de la maternelle. Le Belgique et l'Espagne ont investi dans cette structure ces dernières décennies; leurs établissements reçoivent les enfants tôt (dès la naissance en Espagne, à partir de deux ans et demi en Belgique) et recouvrent la totalité des enfants de 3 à 6 ans. Cela vaut moins pour les autres pays y compris la Finlande, le Portugal et les Etats-Unis, malgré les pas faits dans ce sens depuis les années soixante. ${ }^{2}$ Les enfants pouvaient, et peuvent encore, s'inscrire au Portugal dès trois ans, aux Etats-Unis et en Finlande à partir de quatre voire de cinq ans.

\footnotetext{
${ }^{2}$ Notamment aux Etats-Unis après le lancement du vaste programme Head Start destiné essentiellement aux enfants des afro-américains et des hispaniques, fréquentant alors très peu les établissements préscolaires. (Beatty, 1995 : 192-3).
} 
Quoi qu'il en soit, la représentation et le public de la maternelle s'imprime aussi sur la composition sociale de son personnel. "Le jeu éducatif » écrit A. Prost, "n’est donc pas exactement une invention des maternelles : c'est aussi une pratique des classes supérieures, imitée ensuite par la petite bourgeoisie à prétention intellectuelle. Son succès et sa généralisation résultent précisément de la conjonction d'une institution et d'une classe sociale». «ll n'est donc pas étonnant», poursuit-il, «que nombre des agents de cette institution appartiennent à cette classe. (...) Dans la Seine, Ida Berger a trouvé en 1954-1955 que, plus d'une fois sur trois $(35,3 \%)$, le père des institutrices était un cadre moyen ou supérieur, et leur mari, plus d'une fois sur quatre $(27,4 \%)$ un cadre supérieur ou un membre des professions libérales et près d'une fois sur deux (47\%) un cadre moyen. Elle note aussi que les institutrices partagent plus que les instituteurs les activités culturelles de la bourgeoisie cultivée : théâtre, concert, musée. Or ces traits se sont beaucoup accusés depuis vingt ans. En 1973-1974, les institutrices ont une fois sur quatre un père cadre supérieur ou membre d'une profession libérale et plus d'une fois sur trois (37\%) un père commerçant ou cadre moyen. $13 \%$ seulement des institutrices sont d'origine ouvrière, et $13 \%$ viennent d'une famille d'employés» (1981, p. 107).

Similaire semble être la situation au Japon quant à la composition sociale des institutrices. "Preschool teaching in Japan», note L. Peak, «is a relatively prestigious job for young women. The salary is comparable to that received by college educated women elsewhere in the economy. (...) It is the third most popular course of study at the juniorcollege level, after literature and home economics. (...) It attracts energetic, confident, middle-class and upper middle class young women who typically devote themselves to teaching until they retire from outside employment when their first child is born» (1991, p. $51)$.

\section{Esquisse historique de la maternelle en Grèce}

Concernant la maternelle en Grèce nous distinguons, suivant sa diffusion, son public et ses objectifs pédagogiques, quatre périodes.

I. La première va de l'apparition des premiers établissements préscolaires, aux alentours de 1830, à la découverte et la réception de la pédagogie frœbélienne à la fin des années 1860 
et au début des années $1870 .^{3}$ II s'agit de la période des premiers tâtonnements pédagogiques pendant laquelle voit le jour un nombre très réduit de maternelles, essentiellement privées.

Les premiers à se lancer dans l'éducation des petits enfants sont les missionnaires, protestants en premier lieu, qui fondent des «écoles enfantines». Leur activité suscite la réaction de l'église orthodoxe et des intellectuels qui forment l'Association des Amis de I'Education. Cette rivalité promeut la maternelle et lui confère ses deux premières caractéristiques: sans que les enfants de condition modeste soient exclus, elle s'adresse surtout aux enfants de la «bonne» société; la rivalité, d'autre part, stimule la quête de nouvelles pédagogies.

La perception de la maternelle comme établissement pédagogique se renforce lors des années 1870. L'Association traduit les textes de Pape-Carpantier et les premiers textes sur la pédagogie froebélienne voient le jour. Cette activité va de pair avec un intérêt croissant pour l'enfance dans la littérature grecque et les beaux-arts. Le romancier et essayiste Léon Mêlas dans un commentaire, qui rappelle des textes contemporains des organismes internationaux, écrit en 1871. "L'idée salvatrice de la maternelle fut conçue par notre ingénieux Platon dans ses Lois. Son idée fut adoptée et mise en œuvre presque partout en Europe occidentale». "Nous sommes convaincus», poursuit-il, "que si des maternelles avaient été instituées depuis un certain temps dans toutes les petites villes et les villages de l'Europe occidentale et la fréquentation des enfants y était obligatoire, l'Europe n'aurait pas présentée le sinistre spectacle des bandits, des incendiaires et des assassins (...)» (p. 187, p. $\left.\kappa \beta^{\prime}\right)$.

L'introduction de la pédagogie froebélienne en 1880, notamment par Aikaterini Laskaridou (Kagiadaki, 2009) corrobore l'image de la maternelle comme institution pédagogique. Cette image est confortée par la demande accrue du métier de l'institutrice au cours des années 1870 . Or, cette demande est à l'origine de la mutation de la maternelle d'institution pédagogique en petite école au service d'un Etat-nation en construction. De fait, cette demande est alimentée par la montée des nationalismes dans les Balkans, suite à I'autonomisation, en 1870, de l'Eglise Bulgare du Patriarcat de Constantinople.

Dans ce contexte, la maternelle est appelée à fonctionner comme moyen de création et de diffusion de symboles aux nations naissantes (Moumoulidou, 2002). Cela se reflète sur leur programme centrée sut l'apprentissage de la langue et de l' "histoire».

\footnotetext{
3 Rappelons que pendant cette période prolifèrent en Europe, notamment dans les grandes villes, des institutions, qui s'occupent de la garde des enfants laissés pour compte. Luc, 1997: 15-38.
} 
Logiquement, les maternelles dans les communautés grecques de l'empire Ottoman se multiplient, alors que dans le Royaume grec sont rares.

II. La deuxième période débute dans les années 1870 avec l'adoption de la pédagogie de Fröbel et clôt en 1922. La maternelle fut institutionnalisée en 1895 par un gouvernement conservateur. ${ }^{4}$ Mais cet acte n'a pas été suivi des effets espérés. En revanche, la maternelle se transforme d'institution pédagogique en moyen par excellence au service de l'irrédentisme grec visant avant tout à l'apprentissage de la langue et de l'histoire dans les régions de l’empire Ottoman considérées comme grecques.

Cette visée se répercute sur la politique éducative envers la maternelle et son contenu pédagogique à l'intérieur du pays. En fait, l'État s'abstient de toute action en la matière de sorte que les maternelles sont peu nombreux, privées ou municipales. Par ailleurs, elle s'adresse de plus en plus aux enfants d'origine modeste. Ainsi se produit un déplacement d'orientation: d'institution pédagogique, la maternelle devient établissement chargé de la garde des petits enfants nécessiteux.

III. La question de la maternelle rebondit de nouveau en 1913, à la suite des guerres balkaniques, et en 1922, après la dite catastrophe d'Asie Mineure, qui ont comme conséquence l'augmentation de la population du pays de près de trois millions nouveaux habitants. Depuis, et jusqu'au début des années 1970, la maternelle est appelée de nouveau à servir la nation en socialisant les enfants nécessiteux et en leur apprenant la langue. En fait, les multiples problèmes économiques et sociaux qui découlent de la présence des nouveaux habitants défient l'autorité étatique. Encore une fois, comme d'ailleurs dans bien des pays, ${ }^{5}$ la maternelle, se charge de la diffusion de la langue standardisée et des symboles communs sur l'ensemble du territoire national (Kleanthous-Papadimitriou: 2004, pp. 41112).

La maternelle est alors vidée de son contenu éducatif, elle devient un instrument $d$ 'assimilation et d'homogénéisation. Cette conception est consacrée institutionnellement par la réforme de 1929, mise en œuvre par les Libéraux. Elle lui confère deux objectifs : la

\footnotetext{
${ }^{4}$ Révélateur du temps, le texte législatif est une copie de la loi Goblet alors que la pédagogie est celle de Fröbel (Kiprianos, $2007:$ 89-90).

${ }^{5}$ Les exemples sont nombreux: aux Etats-Unis à la fin du XIXe siècle (Cummings 1984: 283-310), au Japon vers la fin des années 1870 par réaction à l' "occidentalisation " (Wollons, 2000: 123), la Turquie en 1908 après la révolution des Néo turcs et en 1923 lors de l'instauration de la République par Mustafa Kemal (Fortna, 2000: 262 265).
} 
diffusion de la langue et la "socialisation» des enfants "de la rue», ce qui en fait signifie secourir les familles en difficulté. Cela se reflète dans l'horaire restreint de son fonctionnement mais aussi dans le contenu des matières dispensées dans les Ecoles Normales des Institutrices (Vaos, 2000, pp. 153-67). Bref, la maternelle devient une école gardienne au service de la nation.

Dans les faits, la maternelle se limite essentiellement au premier objectif, l'apprentissage de la langue en raison de sa diffusion très sélective. Toutes les écoles de formation des institutrices, sept au total, hormis une, se trouvent dans la dite Grèce nouvelle, i.e. les régions rattachées en 1913. Parallèlement sont créées, à un rythme plus soutenu dans un premier temps, les premières maternelles publiques. Elles seront 445 (et 56 privées) l'année scolaire 1928-29 et 898 en 1959-60 et toutes, à peu d'exceptions près, dans la Grèce nouvelle (Charitos, 1996).

IV. La quatrième période s'étend de 1974 à aujourd'hui. Vers la fin des années 1950 la question de la maternelle resurgit. Les signes innovateurs sont essentiellement exogènes et proviennent de deux vecteurs, de l'OMEP (Organisation Mondiale pour l'Education Préscolaire) et les Organismes Internationaux notamment l'UNESCO. La première, créée en 1948, organise son Vle Congrès, en 1956, à Athènes (O.M.E.P, 1956) Cet événement sensibilise la ministre des Affaires Sociales, Lina Tsaldari, qui y participe et défend la maternelle. Dans le même sens vont les recommandations des Organisations Internationales aux gouvernements, dont celles de l'UNESCO en 1961 et de l'OCDE en 1973 et en 1980, qui préconisent la diffusion et la réorganisation de la maternelle, perçu comme une institution pédagogique de première importance pour le développement cognitif, social et affectif de l'enfant (Kiprianos, 2007, pp. 180-81).

Le contexte est plus propice à la maternelle après 1974. Dans une atmosphère de radicalisation politique, la maternelle occupe le devant de la scène. Toutefois, malgré sa magnification, la réforme de 1976 n'apporte pas de changements radicaux à la maternelle; elle réduit le nombre des enfants par classe à 30 , elle accroît le nombre des inspectrices, elle prévoit la densification du réseau des maternelles. Ces mesures sont suivies de l'introduction d'un nouveau programme en 1980 et une tentative, non consommée, d'extension de la scolarité des Ecoles d'Institutrices de 2 à 3 ans (Programme de 1980).

La reconsidération de la maternelle conduit à l'adoption d'autres mesures en sa faveur après l'ascension au pouvoir en 1981 des socialistes du PASOK. En 1982 l'Ecole des 
Institutrices est reconnue comme établissement universitaire où la durée d'études est de 4 ans. Cette mesure est suivie, en 1985 d'un projet, inspiré de l'exemple suédois, prévoyant l'unification des deux institutions existantes, des crèches et des maternelles, dans une nouvelle institution, appelée "centre d'enfant», censée recevoir des enfants dès la naissance jusqu'à l'entrée au primaire à six ans (Loi 1566 de 1985, ch. B', art. 3.2).

Le projet fut vite oublié, supplanté par la crise économique. Depuis la maternelle a sombré dans l'immobilisme. A retenir trois mesures aux effets, toutefois, relativement réduits: I'institution en 1997 de la dite "maternelle de plein jour», qui consiste en une prolongation facultative de l'horaire jusqu'à 16 heures, et de l'abaissement, en 2004, de l'âge de la scolarité obligatoire à 5 ans. Ces deux mesures modifient peu le statut de la maternelle, en dehors du fait que la première, par la prolongation des horaires, a augmenté la demande des institutrices. Plus importante est la troisième mesure: un effort plus soutenu sur le plan pédagogique qui semble produite plus d'effets à long terme. II pourrait être décrit, dans les termes d'E. Plaisance, comme le passage du modèle productif, centré sur la «connaissance» à l'expressif, reposant sur l' «expression de sa propre personnalité».

\section{Les instituteurs: demande et composition sociale}

Nous avions distingué quatre périodes. D'abord la maternelle s'adresse à tous les enfants, essentiellement ceux de condition aisée. Ensuite, elle s'adresse aux enfants de condition modeste et après 1922 prioritairement aux enfants des familles qui ne maitrisent pas la langue grecque. Enfin, depuis quelques décennies, elle s'adresse à tous.

Ce schéma, doit être envisagé avec précaution. Jusqu'en 1974, le taux des enfants inscrits à la maternelle est trop bas pour que nous puissions nous prononcer avec assurance sur son public. En fait, jusqu'en 1913 les enfants en maternelle s'élèvent à quelques centaines. Un demi-siècle plus tard ce taux se situe entre 5 et $10 \%$ des enfants de 4 à 6 ans, et ne concerne que ceux du Nord du pays, la Macédoine au premier chef. En dépit de la hausse constante de la fréquentation de la maternelle (elle recouvrait en 2005-6 56,1 \% des enfants de 4 à 5 ans et 83,3\% de 5 à 6 ans) (Eurydice, 2009, p. 67), la question de sa composition sociale n'est pas résolue. Bien que nous n'ayons pas de donnes précises il semble que la demande est satisfaite en province mais non dans les villes. ${ }^{6}$

\footnotetext{
${ }^{6}$ Officiellement les maternelles privées attiraient une faible proportion de l'ensemble des enfants de 4 à 6 ans. Cela semble changer en raison de la crise économique actuelle puisque le nombre de ceux qui peuvent financer
} 
Tout cela se traduit dans la demande et la composition sociale des instituteurs, exclusivement femmes jusqu'en 1982. Tout indique qu'ils sont originaires dans leur grande majorité de couches sociales modestes. Cela vaut également, dans un moindre degré, pour aujourd'hui, en dépit de la revalorisation des études en 1982 et de l'amélioration relative, depuis, des perspectives de travail.

Pour mieux nous en rendre compte, rappelons que les instituteurs de l'élémentaire déploraient leur condition sociale et économique depuis la formation de leur confédération syndicale en 1922. Ils déploraient également la déconsidération du métier. Le tableau est encore plus sombre pour les institutrices de la maternelle. De l'étude des archives de l'une des quatre écoles de formation des institutrices de 1965 à 1971, celle d'Alexandroúpolis, ressort que 2 sur 3 sont d'origine agricole et 6 sur 10 sont originaires d'une petite commune. L'extension du réseau des maternelles et la demande croissante en institutrices par la suite modifie la composition des étudiantes quant au lieu de leur provenance mais pas quant à leur condition sociale (Karafillis \& Kiprianos, 2012).

La perception de soi des institutrices des maternelles semble changer depuis 1982. Cela est tangible dans les rapports d'inspection. De l'analyse des rapports annuels d'activité de six inspectrices dans la Grèce du nord, durant la décennie 1985-1995, en résulte un ton plus positif. Bien que ces rapports soient des bilans d'activités et aient été rédigés dans un dessein plutôt de mise en avant de soi, ils affichent une volonté de participation active dans l'éducation de l'enfant, voire dans la vie locale (Zafeirakou, 1997).

Il en va de même de la composition sociale des institutrices. Depuis 1982 les statistiques de l'éducation les incluent dans les facultés des sciences sociales et humaines. Ainsi nous ne disposons pas de données sur leurs origines sociales. Pour nous faire une idée nous avons comparé les données des étudiantes des Ecoles Normales des Institutrices, en 1970 et en 1975, avec celles des étudiants de la Faculté Pédagogique de I'Université de Salonique qui comprend aussi les instituteurs de l'élémentaire et qui, du point de vue des origines sociales du public drainé, est au-dessus de la moyenne nationale. Bien qu'une telle comparaison soit peu appropriée, elle n'est pas inutile car elle nous fournit une première image de l'évolution des origines sociales.

La nomenclature nationale des catégories socioprofessionnelles comporte onze catégories. Laissant de côté la question de la logique de sa construction et de sa cohérence, 
signalons l'écart flagrant entre les origines des institutrices et celles de la «moyenne» des étudiants, écart qui s'atténue quelque peu en 1993. En 1970 et en 1975 la moitié des institutrices est d'origine paysanne, alors que le pourcentage des agriculteurs au sein de la population active se situe en 1971 à $35,7 \%$. Si les familles ouvrières sont plus ou moins analogiquement représentées, très peu nombreuses sont les descendantes des familles de professions libérales et d'employés de bureau, à peine le $1 / 3$ de la «moyenne» des étudiants grecs.

Tableau 2. Origines sociales des institutrices de la maternelle en \% (1970-1993)

CSP

$1970 \quad 1975$
Etudiantes des Ecoles
Normales
d'Institutrices

1993

Etudiants de la Faculté Pédagogique de Salonique

\begin{tabular}{|c|c|c|c|c|c|c|}
\hline Professions Libérales & 3,6 & 3,9 & 10,1 & 11,1 & 10,9 & 24,8 \\
\hline Cadres administratifs & 0,9 & - & -- & 1,7 & 1,0 & 1,1 \\
\hline $\begin{array}{l}\text { Employés de bureau et } \\
\text { assimilés }\end{array}$ & 4,9 & 6,5 & 13,4 & 13,8 & 15,3 & 18,6 \\
\hline $\begin{array}{l}\text { Commerçants et } \\
\text { vendeurs }\end{array}$ & 9,4 & 9,6 & 12,2 & 12,8 & 12,9 & 12,6 \\
\hline $\begin{array}{l}\text { Agriculteurs, pêcheurs } \\
\text { et forestiers }\end{array}$ & 50,9 & 46,2 & 21,3 & 24,8 & 23,7 & 7,9 \\
\hline Ouvriers et artisans & 23,2 & 25,1 & 27,9 & 18,6 & 21,9 & 22,6 \\
\hline Personnels de service & 2,7 & 3,7 & 2,4 & 3,8 & 4,7 & 4,7 \\
\hline Armée et police & -- & 0,3 & 1,7 & 1,5 & 1,8 & 2,5 \\
\hline Retraités, rentiers, & 4,5 & 3,1 & & 8,7 & 6,5 & 5,1 \\
\hline Chômeurs & & 0,3 & & & 0,3 & 0,3 \\
\hline Non déclarés & -- & 1,7 & & 3,1 & 1,0 & 7 \\
\hline
\end{tabular}

Source: Statistiques de l'enseignement de 1970, 1971, 1975 et 1993. http://dlib.statistics.gr/portal/page/portal/ESYE.

Les proportions se modifient quelque peu en 1993. Suite à la diminution rapide de la population agricole $(17,4 \%$ en 1991), les instituteurs des maternelles d'origine agricole sont en baisse mais leur pourcentage est nettement au-dessus de la moyenne. Cette fois sont surreprésentés les étudiants d'origine ouvrière, qui se situent nettement au-dessus de la moyenne mais près du poids de leur catégorie sur l'ensemble de la population. Enfin, bien que les professions libérales et les employés de bureau avoisinent leurs pourcentages respectifs au sein de la population, elles sont nettement au-dessous de la moyenne des étudiants, et, bien entendu, encore 
plus des facultés les plus demandés, comme les facultés de médicine et celles de certains départements d'ingénieurs.

Logiquement, la lente recomposition sociale des instituteurs a du se poursuive jusqu'en 2010 en raison des meilleures perspectives professionnelles suite à l'extension, en 1997, des heures de fonctionnement des maternelles et de la présence d'un nombre croissant d'immigrés. En témoignent l'accroissement des candidats au concours annuel d'accès aux départements relatifs et la montée des notes pour s'y inscrire. Eu égard au nombre des enfants et des maternelles, cette tendance était conjoncturelle et elle ne pouvait pas durer. Toutefois, la modification, fut-elle partielle, de la composition sociale du corps des instituteurs n'est pas sans importance et nous invite à réfléchir sur son sens et sur ses implications potentielles sur la représentation de l'institution et sa valeur sociale.

Sans aborder ici la question, rappelons que cette modification n'est pas le fait de la diffusion des valeurs sociales d'un groupe social, d'habitude des couches moyennes, ou d'une nouvelle représentation collective de l'enfance; elle est plutôt le résultat des effets conjoints d'une certaine représentation de l'enfance et de la maternelle chez une partie réduite de la société, notamment des enseignants et certaines professions gravitant autour l'enfant, et des mesures institutionnelles allant dans le sens de la revalorisation du métier. Or, aucune des deux causes ne se révélait suffisante pour le changement de la maternelle, fait qui explique pour une large part son stationnement actuel.

\section{Conclusions}

Le métier d'institutrice initialement et d'instituteur ensuite a été formé lentement et non sans heurts. L'enthousiasme des premiers pas, notamment pendant les trois dernières décennies du XIXe siècle, n'a pas été suivi d'effets attendus quant à la diffusion de la maternelle. En revanche, la maternelle s'est transformée depuis et jusqu'au début des années 1970 d'institution pédagogique en instrument au service de l'unité nationale. A cette première tâche s'est ajoutée pendant la même période, notamment après 1922, une deuxième de «socialisation».

Dans ce contexte les premières jeunes institutrices perdent l'auréole de l'héroïne pour devenir des employés d'une institution gardienne. Quoi que cela puisse signifier dans 
une société agraire où peu de femmes sont salariées, elles ont deux caractéristiques majeures: Elles sont, dans leur majorité écrasante, des filles d'origine paysanne; et elles sont d'assez bonnes élèves.

La prolifération des maternelles après les années 1970, la revalorisation de leur formation depuis 1984 et l'ouverture aux garçons et en même temps sa re-transformation en institution pédagogique modifient la situation. Le métier se valorise fait qui se reflète sur sa représentation et, par là, sur sa demande et sa composition sociale. Bien qu'il demeure encore, dans une très large mesure, féminin, les institutrices ne sont plus socialement ce qu'elles étaient dans le passé. Sans que leur composition sociale s'approche de celle de leurs collègues en France ou au Japon elle se modifie. II attire les enfants d'origine agricole et ouvrière mais aussi et de plus en plus des couches moyennes. II ne serait pas erroné si nous y repérions les signes du passage inachevé du modèle productif au modèle expressif. 


\section{OUVRAGES ET ARTICLES CITES}

Beatty, B. (1995). Preschool education in America.The culture of young children from the colonial era to the present. New Haven and London: Yale University.

Bernstein, B. \& Young, D. (1973). Social class differences in conceptions of the uses of toys. In B. Bernstein (Ed.), Class, Codes and Control (vol.2, pp.13-23). London \& Boston: Routledge \& Kegan Paul.

Bruner, J. (1996). The Culture of Education. Cambridge, London: Harvard University Press.

Chamboredon, J.C. \& Prévot, J. (1973). Le 'métier d'enfant'. Définition sociale de la prime enfance et fonctions différentielles de l'école maternelle. Revue Française de Sociologie, XIX, 295-335.

Charitos, X. (1996). La maternelle grecque et ses racines. Contribution à I'histoire de l'éducation préscolaire. Athènes: Gutenberg (en grec).

Commission Européenne (1995). L'importance de l'éducation préscolaire dans l'Union européenne. Un état de la question. Luxembourg.

Décret présidentiel 476 relatif au programme analytique de l'école maternelle. Journal du Gouvernement, 31-05-1980, (132).

Eurydice, (2009). Early Childhood Education and Care in Europe: Tackling Social and Cultural Inequalities, Education, Audiovisual and Culture Executive Agency (http://www.eurydice.org).

Fortna, C. (2000). The Kindergarten in the Ottoman Empire and the Turkish Republic. In R. Wollons (Ed.), Kindergartens and Cultures. The Global Diffusion of an Idea. New Haven and London: Yale University Press. 251-273.

Zafeirakou, A. (1997). Recherche et évaluation de la première éducation scolaire : le cas des maternelles dans les préfectures d'Evros et de Rodopi. Rapport non-publié. Université Démocrite de Thrace, Département Pédagogique du Préscolaire.

Kagiadaki, A. (2009). L'école maternelle grecque et les pédagogies européennes (18301936). Thèse de Doctorat des Sciences de l'éducation, Université de Rouen. 
Karafillis, Th., Kiprianos, P. (2012). Opportunités professionnelles, image sociale et choix d'études: Les institutrices de la maternelle lors des années 1960. Nea Paideia, vol. 141: 88-104 (en grec).

Konrad, F.M. (2004). Der Kindergarten. Seine Geschichte von den Anfangen bis in die Gegenwart. Freiburg im Breisgau: Lambertus.

Kiprianos, P. (2007). Enfant, Famille, Société. Histoire de l'éducation préscolaire. Athènes : Gutenberg (en grec).

Kleanthous-Papadimitriou, M. (2004). «Débat». In Conférence des directeurs et des directeurs adjoints des Académies des enseignants du primaire et de la maternelle. Athènes: KEE, 404-421 (en grec).

Liang, X. (1998). Cultural and economic factors influencing children's preschool attendance. New York \& London: Garland Publishing.

Loi 1566 de 1985, Structure et fonctionnement de l'éducation primaire et secondaire et autres règlements, Journal Officiel, № 185, 30-09-1985.

Luc, J.N. (1997). L'invention du jeune enfant au XIXe siècle. De la salle d'asile à l'école maternelle. Paris: Belin.

Mêlas L. (1871). Manuel Pédagogique à I'usage des Enseignants, Athènes: Imprimerie de Vlastos (en grec).

Moumoulidou M. (2002). La moralisation du peuple au moyen de l'éducation préscolaire dans la maternelle du XIXe siècle. In S. Bouzakis (éd.). Thèmes d'actualité d'Histoire de l'Education. Actes de la 1ère conférence de l'Histoire de l'Éducation. Athènes: Gutenberg, 313-326 (en grec).

Office Statistique Grec (Elstat) (2014). Communiqué du 13 Octobre 2014, http://www.statistics.gr/portal /page/ portal/ESYE/BUCKET (en grec).

O.M.E.P. (1956). Report of the 6th World Conference Held at Parnassos, Athens, 10th to 16th of September 1956. 
Peak, L. (1991). Learning to go to school in Japan. The transition from home to preschool life. Berkeley, Los Angeles, London: University of California Press.

Plaisance, E. (1986). L'enfant, la maternelle, la société. Paris : PUF.

Prost, A. (1981). L'école et la famille dans une société en mutation 1930-1980. Paris: Nouvelle Librairie de France.

Vaos, A. (2000). Éducation esthétique dans l'Éducation Grecque. Revue Historique. Approches dans l'Enseignement de l'Art. Athènes : Ellinika Grammata (en grec).

Statistiques de l'enseignement de 1970, 1971, 1975 et 1993. http://dlib.statistics.gr/portal/page/portal/ESYE/

Wollons, R. (2000). The Missionary Kindergartens in Japan. In R. Wollons (dir.). Kindergartens and Cultures. The Global Diffusion of an Idea (pp. 113-136). New Haven and London: Yale University Press. 